\title{
User-Oriented Rule Management for Event-Based Applications
}

\author{
Hannes Obweger, Josef Schiefer, \\ Martin Suntinger, Peter Kepplinger \\ UC4 Senactive Software GmbH \\ Prinz-Eugen-Straße 72, Vienna, Austria \\ \{firstname.secondname\}@uc4.com
}

\author{
Szabolcs Rozsnyai \\ IBM Thomas J. Watson Research Center \\ 19 Skyline Drive, Hawthorne, New York
}

srozsny@us.ibm.com

\begin{abstract}
Event-pattern rules are the foundation of Complex Event Processing (CEP) applications. Yet, despite the vast potential CEP offers for agile business applications, its practical relevance rises and falls with the manageability within the organizational framework conditions of an enterprise. In this paper we present a novel rule-management framework for the event-based system UC4 Decision. It caters to the needs of power users as well as business users: Power users model infrastructural rules based on visual decision graphs and a dedicated expression language. Business users compose rule logic in a simplified web interface from abstracted, configurable building blocks.
\end{abstract}

\section{Categories and Subject Descriptors}

C.2.4 [Computer-Communication Networks]:

Distributed Systems.

\section{General Terms}

Management, Design, Human Factors.

\section{INTRODUCTION}

Complex Event Processing (CEP) enables real-time monitoring of business incidents and automated, event-driven decision making. Event-pattern rules - which Luckham called "the foundation for applications of CEP" [14] - may be considered as decision logic in the form "if situation $x$ occurs in the event stream, then generate response $y$ ". For example, in the online-gambling domain, an exemplary rule could be defined as follows: "If a user shows suspicious betting behavior, then notify fraud department". In a broader sense, rules express the business logic to identify key activities occurring in the business environment from captured event data and the tactical actions to be taken in response.

Despite this definition, we observed that in many practical applications, event-pattern rules rather serve as multi-purpose toolkits to accomplish tasks at different granularities, being applied for the high-level business logic of an application, but also for the lowerlevel (pre-)processing logic required for integrating this business logic with underlying source systems. In particular, these tasks include (cf. [10][14]):

Permission to make digital or hard copies of all or part of this work for personal or classroom use is granted without fee provided that copies are not made or distributed for profit or commercial advantage and that copies bear this notice and the full citation on the first page. To copy otherwise, or republish, to post on servers or to redistribute to lists, requires prior specific permission and/or a fee.

DEBS'11, July 11-15, 2011, New York, New York, USA

Copyright 2011 ACM 978-1-4503-0423-8/11/07...\$10.00.
- Filtering - reducing the overall set of events to be processed by an event-processing agent to those events that are actually relevant for the given processing task, e.g., removing erroneous or incomplete data (processing logic).

- Transformation - conditional enrichment and/or adaptation of events, e.g., setting an event attribute $a$ based on the value of another attribute $b$ (processing logic).

- Aggregation - aggregating lower-level events to higherlevel complex events based on semantic, temporal and sequential conditions; e.g., create a complex "Bet Finished" event from a sequence of related "Bet Placed", "Sports Event Ended", and "Bet Won" events (processing logic).

- Situation detection - triggering actions in the source systems in response to the occurrence of defined event situations, e.g., blocking an account in response to fraudulent betting behavior (business logic).

Depending on their particular function within an event-based system, event-pattern rules are associated with different user groups in an enterprise, each having specific skills, responsibilities and competences. Existing approaches to rule management for event-based applications - i.e., the overall set of tools and workflows provided for the creation, application and administration of event-pattern rules - tend to disregard the above-described diversity of event-pattern rules, though. This reflects in rulemanagement systems offering the level of control and expressiveness to cater to the needs of modeling sophisticated processing logic, but neglecting the needs of business users for simple management and control over the business logic. As a consequence, business users are either required to have extensive technical skills or rely on technical experts from the IT department to implement changes in the business logic (Figure 1a).

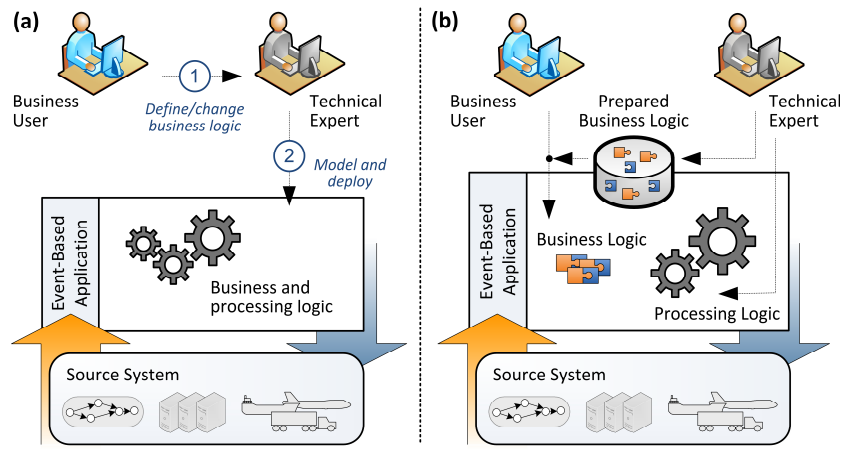

Figure 1. Comparison of rule-management workflows in existing systems (a) and the proposed approach (b). 
In this paper, we present a novel approach towards rule management for the Complex Event Processing system UC4 Decision. ${ }^{1}$ We propose a framework of tools and workflows for modeling processing logic and business logic based on a unified rule-evaluation model. Yet, comparing to other existing approaches the degree of control and complexity is adjusted to the needs and skills of the different user groups in an enterprise: Developers and technically-skilled domain experts model rules by means of visual decision graphs and a specific expression language yielding highest expressiveness. Business users combine and deploy rules from predefined building blocks of business logic in a simplified, wizard-based interface. The resulting change in the rule management workflow is depicted in Figure 1b.

We claim that such an approach eases the administration of business rules, facilitates authorization and security control for business critical rules and reduces the number of errors associated with rule modeling.

\subsection{Conceptual Foundations}

As a conceptual foundation for our approach to rule management, we propose a differentiation of event-pattern rules by the general function of a rule, into

\section{a. infrastructural rules and \\ b. Sense-and-Respond $(S \& R)$ rules.}

Both kinds of rules are equivalent regarding their basic semantics: In both cases, actions are triggered in response to detections of an event pattern in underlying streams of events. They differ, however, in the way they are created, applied and administrated in the proposed rule-management framework. Figure 2 shows the roles of infrastructural and S\&R rules in an event-based application.

Infrastructural rules, on the one hand, include all rules that serve as an input for other parts of an event-based application, but not respond back to underlying source systems by themselves. Along with an event-type model, an event-service orchestration and possible mechanisms for aggregating event data, the collection of infrastructural rules of an event-based system may therefore be considered the event-based infrastructure for creating an eventbased, near real-time abstraction of the source systems: All relevant real-world incidents and state changes are then accessible at a proper level of granularity, via accordingly pre-processed events and/or aggregated event data.

Being part of a so-defined integration layer between the realworld business environment and the actual decision making, infrastructural rules are critical to the overall functioning and performance of an event-based application, and must operate in full accordance with the other elements of an event-based infrastructure. In the proposed model, we thus provide for infrastructural rules to be managed by technically-versed power users of a CEP framework: These so-called system operators model infrastructural rules in a single, comprehensive model (as so-called rule definitions) and directly apply them at the respective ruleexecution units in an event-based application. The proposed workflow especially focuses on efficiency, transparency and immediacy and strives to minimize administrative overhead as would arise from a more abstracted approach.

\footnotetext{
${ }^{1}$ http://www.uc4.com/products/uc4-automation-platform/uc4decision.html
}

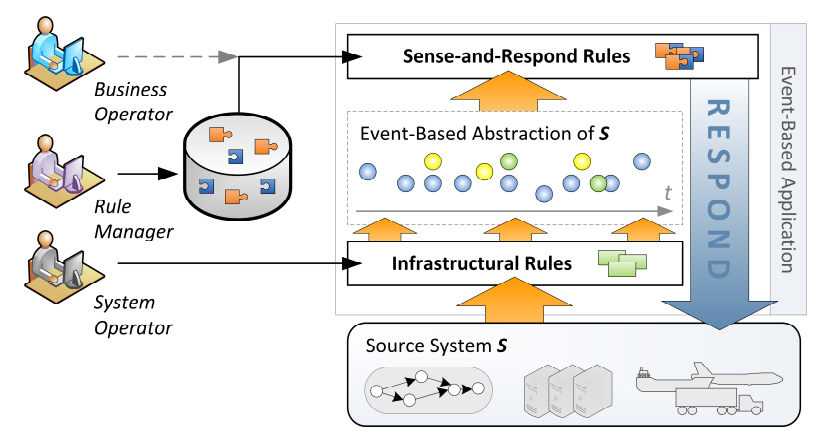

$\square$ Rule Definition $\square$ Pattern Definition $\asymp$ Action Definition

Figure 2. Infrastructural vs. Sense-and-Respond rules.

Sense-and-Respond $(S \& R)$ rules, on the other hand, include all rules that do not again serve as an input for other parts of an event-based application, but directly or indirectly respond back to the source system. Setting up on an up-and-running event-based infrastructure (including the infrastructural rules of an application), S\&R rules therefore cover the actual decision making for supervising and steering an underlying business environment: $\mathrm{S} \& \mathrm{R}$ rules continuously monitor the given event-based abstraction for relevant business situations, and, in response to such situations, trigger respective actions in the source systems.

Eventually feeding back to underlying source systems, appropriately-defined $S \& R$ rules are critical to the correct operation of an observed business environment. However, creating and applying such rules "from scratch" would not only require the domainspecific expertise of customer-side representatives, but would also force these users to get acquainted with the diverse mechanisms for creating and applying such rule logic as part of an event-based application. We therefore propose a two-step workflow for managing S\&R rules:

In the first step, we allow for technically adept and well-trained domain experts - so-called rule managers - to define a catalog of configurable, easy-to-use "building blocks" of a.), encapsulated pattern-detection logic (pattern definitions), and b.), encapsulated reaction logic (action definitions). These buildings blocks strictly abstract from underlying complexity; i.e., from an end-user point of view, the prepared catalog of event-processing logic appears as a collection of relevant business situations (e.g., "suspicious user behavior") and possible actions (e.g., "send an email to the fraudprevention department").

In the second step, appropriately-configured buildings blocks are then assembled to concrete S\&R rules in the form "if event situation, then action( $s)$ " depending on the actual controlling requirements of the underlying business environment. The creation and maintenance of the concrete steering logic still requires domainspecific, detailed knowledge of the source system; however, it fully abstracts from the event-based foundations of an application. Also, due to S\&R rules" "read-only" access to the underlying event stream of real-world incidents, they may be added, changed and removed without having users to consider any side-effects to other parts of the application. The process of instantiating concrete S\&R logic from prepared building blocks is performed socalled business operators, which will usually be domain experts with little or no technical expertise. 
Covering both processing logic and business logic, the overall process of creating a full-fledged, up-and-running event-based application can now be summarized as follows:

- System operators establish an event-based infrastructure, including all infrastructural rules of an application.

- Rule managers create a catalog of "building blocks" of encapsulated pattern-detection logic and reaction logic.

- Business operators assemble these building blocks to concrete Sense-and-Respond rules.

The remainder of this paper is structured as follows: In Section 2, we present related work. Section 3 gives a short introduction to the event-processing facilities of UC4 Decision, which serves as the basis for our approach. Section 4 and Section 5 discuss the proposed approaches to infrastructural rule management and sense-and-respond rule management in greater detail. Section 6 discusses the implementation of our framework with UC4 Decision. Section 7 concludes this paper and provides a short discussion of the experiences we made with the presented framework.

\section{RELATED WORK}

Since introduced to a wider community by David Luckham and his seminal work on "The Power of Events" [14], Complex Event Processing has inspired numerous projects of both academic (e.g. [1][3][7][17][21]) and commercial nature (cf. [11]) as well as in the Open Source community [9].

Albeit more and more end users are concerned with the setup and maintenance of event-based applications in their daily work, discussions on CEP have long focused on operational features such as expressiveness and performance (cf. [12]), and there is little work on rule management in event-based systems. Luckham [15] lists rule management as one of the challenges for future CEP systems. GRUVe [22] is a four-phase methodology for managing complex event patterns throughout their lifecycle. It primarily builds upon the reuse of existing event patterns, which is supported by a semantic event-pattern model. We agree with the authors that reuse is of paramount importance for efficient rule management. However, we believe that one common workflow for both business and power users will in many cases lead to restrictions for at least one group of users.

While little work addresses the broader issue of rule management, there is an active discussion on how to make CEP accessible to business users. Etzion and Niblett [10] see the development from programming-centred to semi-technical development tools as one of the emerging directions in event processing. Chandy and Schulte [5] identify the ability to "enable business users to tailor systems to their needs" as a major criterion for the relevance of an event-based system, arguing that a one-size-fits-all specification of events and responses doesn't work. Mismatches between the complexity of event-processing logic and the abilities of potential adopters have already inspired the definition of domain-specific reference models [4] and an approach to automated rule parameter prediction and correction [23]. We support business users with a tailored workflow, where fully-abstracted, configurable building blocks are assembled to rule instances. An alternative, less abstracted workflow is provided for power users.

The presented separation of event-pattern rules into infrastructural rules and Sense-and-Respond rules eventually results in a twolayered architecture for event-processing applications. Layered application models have a long history in CEP. Luckham [14] and Paschke and Vincent [16] discuss layered models for eventprocessing networks (EPNs). MavEStream [6] is a layered architecture that allows integrating continuous query processing and CEP. Kellner and Fiege [13] present the separation of two viewpoints in a CEP application, which facilities a business-oriented, top-down approach based on Key Performance Indicators (KPIs). The authors claim that with their approach "changes in situations to be detected can be handled without affecting the derivation of values for KPIs that are more stable". Similarly, our approach allows changing high-level business logic decoupled from lowlevel processing rules. Eckert and Bry [8] present XChange ${ }^{\mathrm{EQ}}$, a high-level event-processing language differentiating event-pattern rules based on their function within a CEP application into deductive rules and reactive rules. With slightly different syntaxes, the separation intends to make an application more clear. Similarly, we separate rules into infrastructural and S\&R rules. Yet, in contrast to the described approaches, we explicitly associate the layers with different workflows and user groups. This provides not only a clear application structure, but also enables to tailor workflows and user interfaces to the according user groups and achieve a clear separation of concerns.

In the field of business rules, rule management is long recognized as an important component for practical deployments. Business Rule Management Systems (BRMSs) complement business rule engines with rule repositories and rule authoring tools. Most BRMSs aim to provide a rule syntax that is close to natural language. Domain-specific languages (DSLs) are an extension to general-purpose rule languages that can be defined by power users based on the requirements of a given business scenario. To our best knowledge, there is no event-processing language (EPL) in the CEP space that claims to converge to natural language. We believe that this is because of the increased complexity of eventpattern rules in comparison to business rules. Rule templates are partially defined rules with placeholders to be populated by business users. Allowing business users to instantiate arbitrarily complex rule logic, rule templates are particularly suitable for CEP, and have, for instance, been used with AMiT [2]. In our approach, we extend the concept of rule templates and provide templates not only for complete rules, but also for decoupled "building blocks" of rule logic. Such building blocks can then instantiated and assembled to concrete rules by business users.

\section{ARCHITECTURAL BACKGROUND}

UC4 Decision is a commercial CEP platform that is based on Sense-and-Respond Infrastructure (SARI) as originally proposed by Schiefer et al. [20] at DEBS'07. In the following, we give an overview to the general architecture and core event-processing facilities of UC4 Decision; our approach to user-oriented rule management as presented in Section 4 and 5 of this paper will set up on these concepts and extends them towards manageability for the different user groups within an enterprise. For more detailed discussions on UC4 Decision/SARI, its rule language, its approach to distributed event processing and data management, the interested reader may refer to related work [18][19][20].

The central concept of any UC4 Decision application is the eventprocessing map, a user-defined orchestration of event adaptors and event services as sketched in Figure 3. Event adaptors may be considered the actual interface to underlying source systems: Depending on their implementation, event adaptors translate real- 

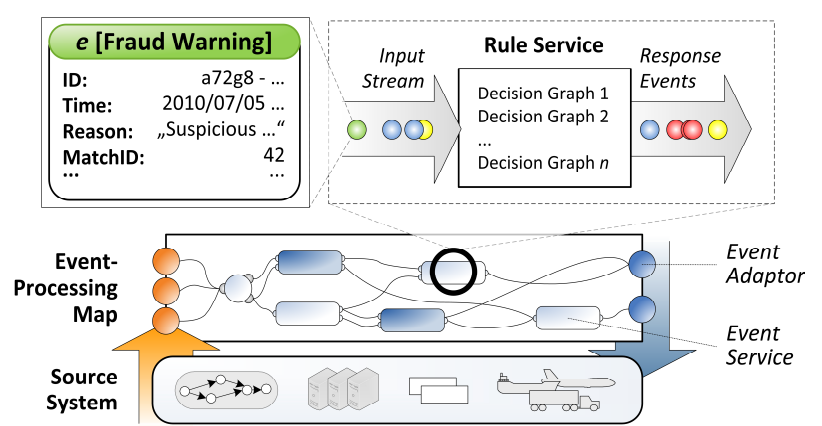

Figure 3. Sense-and-Respond Infrastructure.

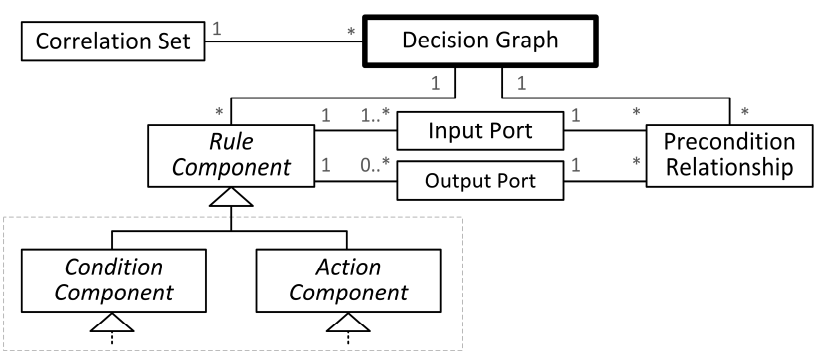

Figure 4. Decision graph meta-model.

world actions (such as a user actually placing a bet in an online gambling platform) into event representations of certain event types, and vice versa. As shown for an exemplary "Fraud Warning" event in Figure 3, events may further characterize signified real-world incidents through collections of event attributes as defined in their event type. Event services receive events from event adaptors or other event services, process them based on implementation-specific logic and respond back to the event processing map. Event services therewith cover the entire event processing of a Decision application; an event service could, for instance, pick from a stream of events those that are relevant in a certain context, publish events to a repository for later analysis, or serve as a hub. Rule services are special event services that allow evaluating sets of decision graphs on incoming event streams.

Decision graphs form the foundation of any pattern detection in UC4 Decision and will serve as a technical basis for both infrastructural rules and $S \& R$ rules as discussed in the remainder of this paper. A decision graph may be considered a directed, acyclic graph of easy-to-understand pieces of rule logic - so-called rule components - such as "the occurrence of an event of type $T$, with certain attribute values" or "the generation of a response event of type $U$ ". Predecessors in the decision graph are then considered as preconditions in the evaluation process: To activate a rule component, an event stream must conform to (at least) one valid path through the decision graph.

Figure 4 shows the meta-model for decision graphs. Depending on its implementation, each rule component has a collection of input ports and output ports: While former allow generally activating a rule component, latter represent possible results of the encapsulated logic. A precondition relationship associates an output port of a rule component $c_{i}$ with an input port of another rule component $c_{j}$. In case of multiple predecessors, a binary input-port operator defines whether all, at least one, or exactly one precondition must be fulfilled in order to activate an input port.

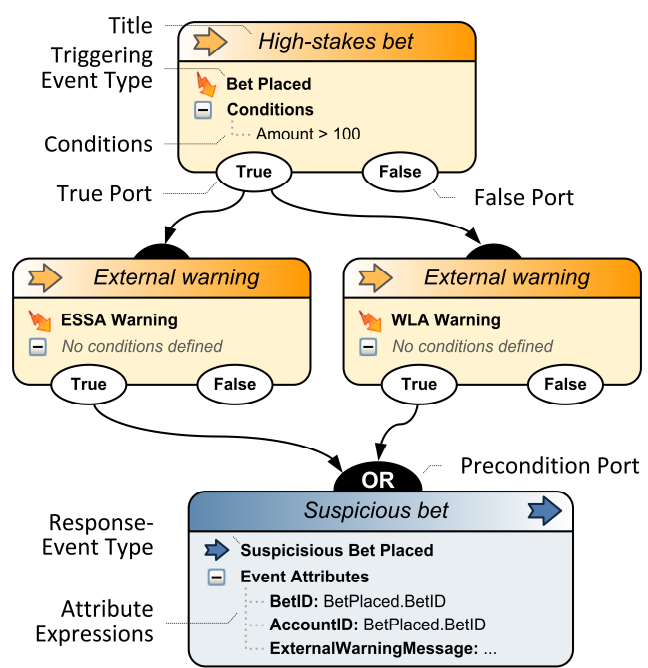

Figure 5. Exemplary decision graph.

Rule components may encapsulate a wide range of eventprocessing logic and make decision graphs a powerful and extensible toolkit for detecting event situations. Rule components may generally be separated into condition components and action components, respectively. Condition components allow modeling the certain characteristics of an event situation by evaluating userdefined expressions on the incoming event stream and activating their output ports depending on the result of that evaluation. Event conditions, for instance, evaluate a Boolean expression on each occurrence of a user-defined event type; output ports are available for "true" and "false". Action components typically serve as the end nodes of a decision graph and encapsulate reaction logic to be executed whenever an event stream conforms to the component's preconditions. Response-event actions, for instance, encapsulate the generation of a certain kind of response event, which is then published to the event processing map.

Figure 5 shows the rendering ${ }^{2}$ of a simple decision graph from the fraud-detection domain, assembled from two event conditions and a response-event action. In the example, high-stake bets (with a bet amount greater than 100\$) are transformed into "Suspicious Bet Placed" events whenever an external warning is retrieved from at least one of two global early-warning systems. Via its event attributes, the response event holds the concerned account and bet IDs as well as warning message. Note that correlation information - e.g., that all events must belong to the same sports event - is defined in a separate model; these so-called correlation sets [19] are referred by the decision-graph model. For accessing elements of the underlying event stream and performing calculations on these data, rule components use a tailored expression language, so-called EA Expressions [18]: In the above example, EA Expressions are used both for formulating a condition on the triggering "Bet Placed" event in the initial "High-stakes bet" component and for addressing event attributes of a preceding "Suspicious Bet Placed" event in the response-event action.

\footnotetext{
${ }^{2}$ Decision-graph renderings as shown in this paper have been optimized for readability.
} 
Business entities eventually allow aggregating the state of durable entities in application-wide, typed data structures. A businessentity instance can be retrieved via a (possibly composite) key and updated and queried via an interface. UC4 Decision currently supports three basic kinds of business entities: Scores are numeric values that are typically used as counters. Base entities and sets allow establishing virtual representations of corresponding realworld structures. While base entities group collections of attributes, sets offer methods for adding and removing items and can then be queried for metrics such as the average sojourn time.

\section{INFRASTRUCTURAL RULES}

In Section 1, we have introduced the notion of infrastructural rules for all rules that prepare data for other parts of an application, but do not by themselves respond back to the underlying source system. In the following, we present our approach to infrastructural rule management and its realization as part of UC4 Decision's event-processing architecture. It is based on the idea of letting power users of an event-processing framework - so-called system operators - define infrastructural event-processing logic in a single, comprehensive model and directly place and enact this logic at respective parts of an event-based application.

We identified the following requirements for an approach to infrastructural rule management

- Expressiveness: Rule-based event processing has proved useful for a wide range of infrastructural issues, including filtering, event enrichment, the creation of composite events and the maintenance of business entities. Infrastructural rules must be expressive enough to accomplish these tasks and establish an appropriate abstraction of underlying source systems.

- Efficiency of use: Infrastructural rules are created and applied by power users of a CEP framework. To facilitate the efficient creation of an event-based infrastructure, a framework shall therefore make infrastructural rule management as immediate, clear and transparent as possible, and focus on these aspects in preference to however-defined abstractions from underlying complexity. Infrastructural rule management shall furthermore minimize any overhead that may arise from administrating rules and their assignment to rule services.

- Full and system-wide access: Being part of the event-based infrastructure of an application, adding, changing or removing infrastructural rules is likely to affect calculations in other parts of the application. A framework shall therefore provide full and comprehensive access to an application, enabling users to efficiently investigate and handle possible side effects.

In the presented framework, system operators model infrastructural event-processing logic as application-wide rule definitions, in parallel and fully integrated with the other elements of an application's event-based infrastructure. Rule definitions may be considered as self-contained, fully-functional pieces of rule logic: Encapsulating event-processing logic of the form "if pattern, then action(s)" in an integrated model that is interpretable to UC4 Decision, rule definitions can directly and without further instantiation steps be applied to incoming event streams. For its enactment as part of a Decision application, system operators eventually assign a rule definition to one or more rule services across the event-processing map of an application. During run time, each rule service then evaluates an independent instance of the represented event-processing logic.

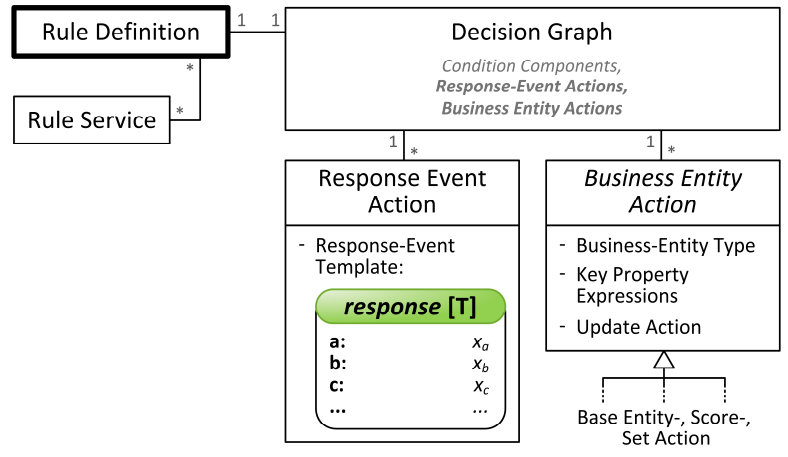

Figure 6. Rule definition meta-model.

UC4 Decision supports the described workflow through a comprehensive, power-user-oriented IDE for event-based applications; in UC4 Decision Modeling Studio, system operators are provided with tools to create and administrate event-processing map, rule definitions, event types and business entities.

Figure 6 shows the meta-model for rule definitions. The key element of any rule definition is a reactive decision graph, i.e., a decision graph that is assembled from condition components and one or more action components. UC4 Decision currently features four types of action components: Response-event components generate a response event based on a user-defined response-event template. The response-event template is defined by an event type $T$, and, for each event attribute $(i, t) \in T$, an expression on the underlying event stream returning a value of type $t$. Base entity-, score- and set-action components allow updating the different kinds of business entities in an application. In all cases, a rule component is defined by a business-entity type, and, for each key property of that type, an expression on the underlying event stream. On the identified business-entity instance, the rule component then invokes a user-defined update function; a rule component could, for instance, increment or reset a score.

A so-defined rule definition clearly implies a tight coupling of pattern-detection logic and response logic, preventing users from reusing rule logic in other contexts. Note, however, that infrastructural rules typically encompasses highly specific logic that is required exactly once in a system and makes sense only "as is". Decoupling the pattern-detection part and the reaction part of an infrastructural rule would therefore cause considerable effort not only for the actual decoupling, but also for the administration of the so-created sub-entities, for little or no gain. Also, using a single decision graph provides a comprehensive view on a rule; this simplifies the creation of complex rule logic and bug-fixing.

\section{SENSE-AND-RESPOND RULES}

In Section 1, we have introduced the notion of Sense-and-Respond $(S \& R)$ rules for all rules that do not prepare data for other parts of an application but directly or indirectly respond back to the source system. In the following, we discuss our proposed approach to $S \& R$ rule management. It is based on the idea of a.), rule managers preparing building blocks of pattern detection and reaction logic, and b.), business operators assembling these building blocks to concrete rules of the form "if pattern, then action(s)".

From the idea of easy to use building blocks, we derived the following requirements for an approach to $S \& R$ rule management: 
- Decoupling of pattern-detection and reaction logic.

- Reusability of building blocks across different use cases, i.e., configurability.

- Ease of use: Performed by business users with limited technical expertise, the administration of $\mathrm{S} \& \mathrm{R}$ rules shall be as simple and fail-safe as possible, and fully abstract from the eventbased foundations of decision making. Users shall neither have to care about the implementation of building blocks nor about the execution of concrete S\&R rules as part of an application.

- Hot deployment: Given a running event-based infrastructure and a set of predefined building blocks, business operators shall be able to work autonomously, generally independent from other users or any kinds of temporal restrictions. As a consequence, an approach to $\mathrm{S} \& \mathrm{R}$ rule management shall support "hot deployment" of rules, i.e., to enact, change and remove rules without having to stop the entire system.

- Security: A rule-management framework shall enable rule managers to clearly define the competences of a given business operator, i.e., to define which building blocks are available to him or her for creating concrete S\&R rules.

In the following, we discuss the key elements of our approach to S\&R rule management. Pattern definitions and action definitions implement the concept of prepared building blocks of pattern detection and reaction logic. Rule instances represent concrete $\mathrm{S} \& \mathrm{R}$ rules assembled from these building blocks. Rule spaces group related building blocks and serve as a basis both for mapping $S \& R$ rule logic to rule services and user rights management.

\subsection{Pattern Definitions}

Encapsulating pattern-detection logic of a form that can be interpreted by UC4 Decision, pattern definitions represent the first category of easy-to-use "building blocks" in the proposed approach to $S \& R$ rule management. Together with their counterpart for reaction logic - so-called action definitions - pattern definitions form the base elements of any concrete S\&R rule: Any rule is, eventually, based on the instantiation of a pattern definition; when an event situation matches the pattern, the associated reaction logic is triggered. In the proposed workflow, both kinds of building blocks are created by technically-versed domain experts - so-called rule managers - based on the general monitoring requirements of an enterprise. As with system operators, rule managers use the Modeling Studio for editing a Decision application; yet, in contrast to the former, rule managers are granted access to pattern and action definitions only.

Figure 7 and Table 1 show the meta-model for pattern definitions along with a concrete example. A pattern definition $p=$ $(I N, O U T, d, t)$ is defined by a collection of input parameters IN, a collection of output parameters OUT, a passive decision graph $d$ and a textual representation $t$. A decision graph is said to be passive if it does not contain any action component but is assembled from condition components and so-called signals only.

Input parameters allow configuring the pattern-detection logic of a pattern definition based on the specific use case in which it is used, without having to change and understand the encapsulated low-level pattern-detection logic. Within the decision graph of a pattern definition, input parameters may be used as typed placeholders in the various expressions of the used rule components; given an integer-typed parameter "Threshold", a condition on "Bet Placed" events could be defined as follows:

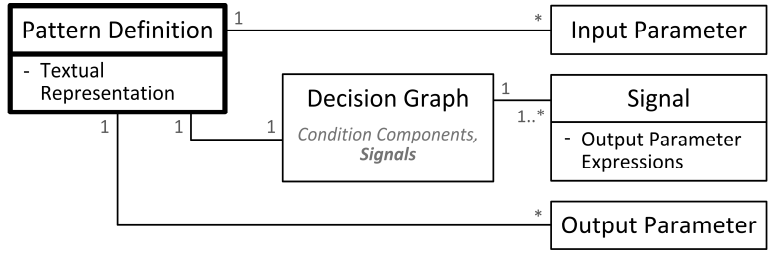

Figure 7. Pattern definition meta-model.

Table 1. Pattern definition example.

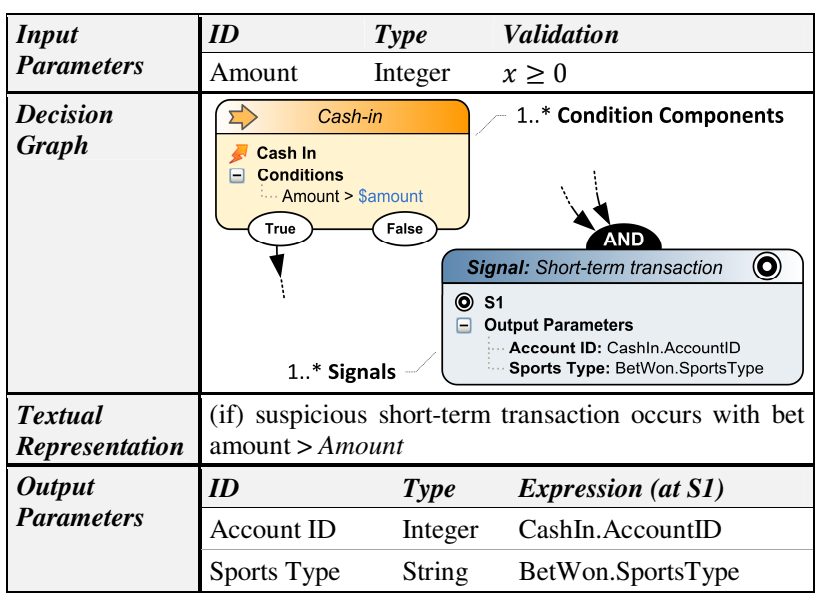

\section{BetPlaced.Amount $>$ \$Threshold}

An input parameter in $=(i, t$, validate $)$ is defined by an identifier $i$, a data type $t$ and an optional validator validate: $t \rightarrow\{0,1\}$. If specified, a validator allows further restricting the set of possible input-parameter values for $i n$; given an input-parameter value $v: t$, validate $(v)=1$ must hold.

Output parameters. In almost any use case, the action part of an event-pattern rule demands access to selected characteristics of the triggering event situation. The extraction of such data requires, however, detailed knowledge of the matched event sequence. Pattern definitions provide for an abstraction of the triggering event situation through the use of output parameters, which enable rule managers to specify those aspects of a triggering event situation that are supposed to be relevant when using the pattern definition in concrete $S \& R$ rules. To integrate the action part with the pattern part of a rule, business operators may then resort to a plain list of typed data fields. In the proposed model, an output parameter out $=(i, t)$ is defined by an identifier $i$ and a data type $t$; the actual value of an output parameter is calculated in the various signals of the pattern definition's decision graph.

Signals. The actual event-processing logic of a pattern definition is defined as a decision graph, which allows evaluating instantiated pattern-detection logic on common rule services. The decision graph of a pattern definition is thereby required to be passive, which means that is must not contain any action components; instead, the specific actions to be taken in response are specified on the level of S\&R rules, by business operators. A decision graph still requires, however, a dedicated class of rule components to signify the detection of a matching event situation. Signals are special action components that abstract from concrete reaction logic and simply notify the detection of an event situation to 


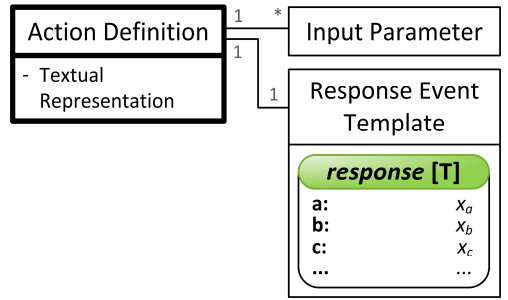

Figure 8. Action definition meta-model.

Table 2. Action definition example.

\begin{tabular}{|c|c|c|c|}
\hline \multirow{3}{*}{$\begin{array}{l}\text { Input } \\
\text { Parameters }\end{array}$} & $I D$ & Type & Validation \\
\hline & Text & String & - \\
\hline & Priority & Integer & $1 \leq x \leq 5$ \\
\hline $\begin{array}{l}\text { Textual } \\
\text { Representation }\end{array}$ & \multicolumn{3}{|c|}{$\begin{array}{l}\text { (then) send alarm with text Text and priority } \\
\text { Priority to fraud department }\end{array}$} \\
\hline \multirow[t]{7}{*}{$\begin{array}{l}\text { Response-Event } \\
\text { Template }\end{array}$} & \multicolumn{3}{|c|}{$\begin{array}{l}\text { Response-Event Type: } \\
\text { com.example.common.Email }\end{array}$} \\
\hline & \multicolumn{2}{|c|}{ Event Attribute } & Expression \\
\hline & To & String & "fraud@example.com" \\
\hline & Subject & String & “Alarm! Alarm!” \\
\hline & Text & String & "Alarm:" + \$text \\
\hline & Priority & Integer & \$priority \\
\hline & $\ldots$ & $\ldots$ & $\ldots$ \\
\hline
\end{tabular}

arbitrary signal listeners. A signal $s=\left(i, X_{\text {OUT }}\right)$ is defined by an identifier $i$ and a collection of output-parameter expressions $X_{O U T}$ for all output parameters in OUT. When a signal is activated, these expressions are evaluated and concrete output-parameter values are calculated. By definition, the decision graph of a pattern definition must contain at least one signal.

Textual representation. Pattern definitions (as well as action definitions) are eventually provided to business operators in the form of a natural-language, textural representation $t$ of the encapsulated event-processing logic, with placeholders for all input parameters. To facilitate a high-level, business-oriented approach to rule creation, the textual representation of a pattern definition would typically describe the real-world business situation that originally caused a matching event situation rather than the exact event sequence by itself; for instance, given a fraud pattern, one would speak of a "fraud attempt in league $x$ " rather than a specific sequence of "Cash In", "Bet Placed" and "Cash Out" events. In Table 1 and 2, input parameters are shown in italic font.

\subsection{Action Definitions}

Action definitions encapsulate pieces of reaction logic and form the counterparts to above-described pattern definitions on the level of easy-to-use building blocks of business logic. In the presented rule-management framework, an action definition may thereby be considered as a blueprint for concrete response events; at run time, such response event then triggers the depicted realworld action in a downstream event service or the source system. For instance, an action "Email to fraud department" could encapsulate the generation of an event of type "Email", with the event attribute "Receiver" set to "fraud@example.com".
Figure 8 and Table 2 show the meta-model for action definitions along with a concrete example. An action definition $p=(I N, r, t)$ is defined by a collection of input parameters $I N$, a responseevent template $r$ and a textual representation $t$. As with pattern definitions, input parameters allow configuring the encapsulated event-processing logic depending on the specific context in which it is used. Within an action definition, an input parameter may now be used as a typed placeholder in the diverse event-attribute expressions of the response-event template; the concrete attribute values of a response event then depend on the input-parameter values as eventually specified in an S\&R rule. The response-event template $r=(T, X)$ defines the general structure of an action definition's event representation. It is defined by an event type $T$ and a collection of event-attribute expressions $X$ for all event attributes in $T$. The textual representation $t$ of an action definition would typically describe its ultimate result (as it would be visible to business operators) rather than its immediate, technical implications; for instance, an action definition that results in an event of type "Email" would be described as "sending an email".

\subsection{Rule Instances}

In the final step of the proposed workflow, pattern definitions and action definitions are eventually presented to business operators, which assemble these blocks to concrete event-processing logic of the form "if patterns, then action(s)". With both kinds of building blocks abstracting from underlying complexity through highlevel, textual representations of the encapsulated event-processing logic, the process of assembling a $S \& R$ rule can thereby be presented to users as assembling a natural-language sentence of the form "if real-world situation, then real-world action(s)", from prepared clauses. Input parameters are seamlessly integrated into the textual representations and can successively be replaced by concrete values during the instantiation process.

For the creation and administration of S\&R rules, UC4 Decision provides business operators with an easy-to-use, wizard-based web interface - the so-called Web Control Center. Figure 10 shows a screenshot from the Web Control Center's central rulecreation wizard: After choosing a pattern definition in the previous step of the wizard, the user may add action definitions to the rule instance by dragging the respective building blocks from a selection panel to the rule structure. Input parameters are rendered as links; on clicking a link, a popup dialog allows setting a constant value or an EA Expression in a type-safe editor.

Figure 9 shows the meta-model for rule instances. A rule instance $r=\left(p, X_{p}, B\right)$ is defined by a pattern definition $p$, a collection of input parameter expressions $X_{p}$ for all input parameters of $p$ and a collection of bindings $B$. Bindings are auxiliary constructs that associate the pattern definition with one or more action definitions; a binding $b=\left(a, X_{a}, C\right) \in B$ is defined by an action definition $a$, a collection of input-parameter expressions $X_{a}$ for all input parameters of $a$ and a collection of conditions $C$.

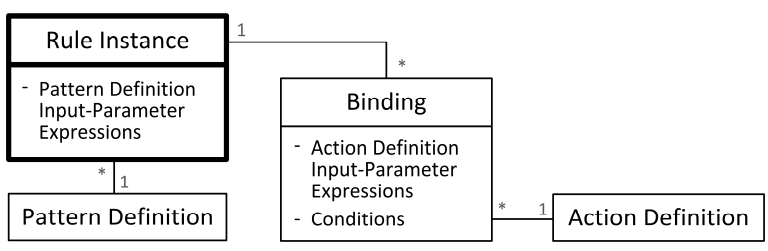

Figure 9. Rule instance meta-model. 

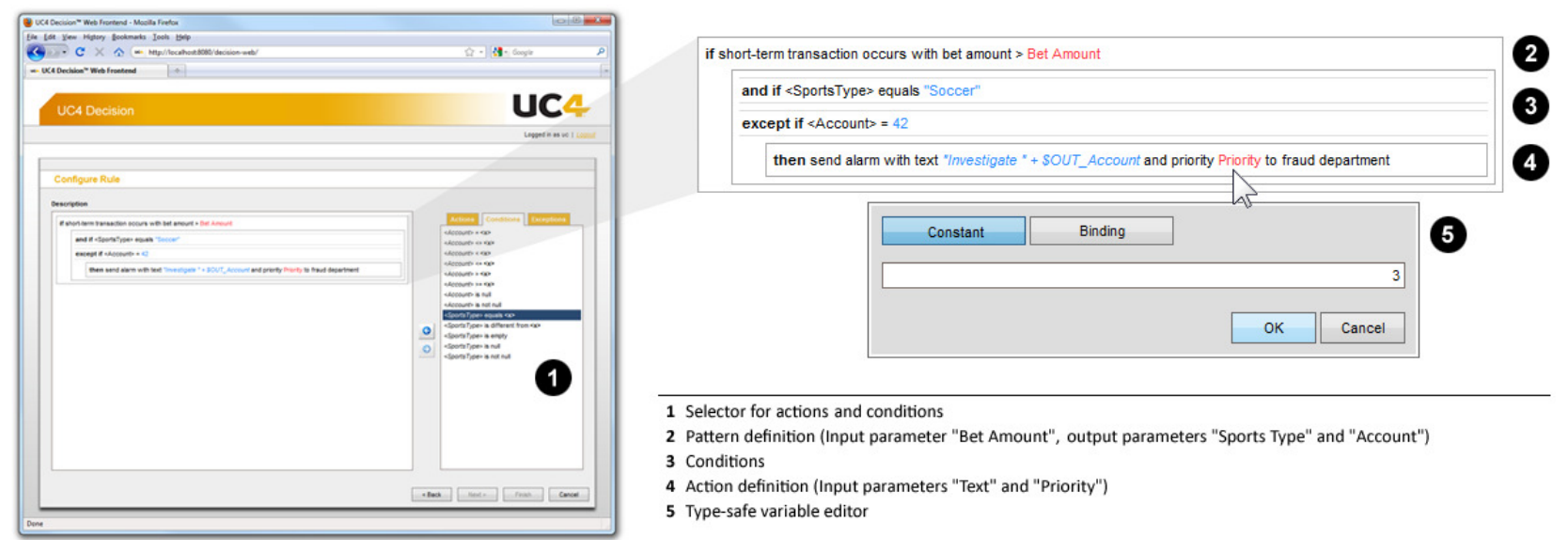

1 Selector for actions and conditions

2 Pattern definition (Input parameter "Bet Amount", output parameters "Sports Type" and "Account")

3 Conditions

4 Action definition (Input parameters "Text" and "Priority")

5 Type-safe variable editor

Figure 10. Creating Sense-and-Respond rules using UC4 Decision Web Control Center

Input parameter expressions define concrete values for the diverse input parameters of the rule's pattern definition and its action definitions, respectively. In case of action definitions, input parameter expressions may calculate such value from the output parameters of the pattern definition. This enables business operators to adapt reaction logic dynamically based on the triggering event-situation instance. Pattern-definition input-parameter expressions, by contrast, are necessarily constant.

Conditions provide a simple mechanism for business operators to further specify pattern-detection logic based on the characteristics of a triggering event situation, in a way that abstracts from the event-based foundations of decision making. A condition may basically be considered is a Boolean expression involving one or more output parameters of the pattern definition, which is evaluated whenever the pattern is detected. Only if all conditions of a binding evaluate to true, the associated action is executed. In the Web Control Center, meaningful conditions are generated based on the output parameters of the chosen pattern definition and can be added by "drag and drop" similar to action definitions.

It is essential to note that rule instances are not directly mapped to rule services; by contrast, such association is established implicitly - in a way that is transparent to business operators - through the concept of rule spaces as discussed in the next section.

\subsection{Rule Spaces}

Rules spaces group the pattern definitions and action definitions of a Decision application (both, in many-to-many relationships) based on the organizational tasks to which they belong; for instance, a rule space "Fraud Detection" could contain eventprocessing logic for detecting different kinds of fraudulent user behavior, notifying the fraud department, and blocking an account automatically. In this way structuring the overall S\&R eventprocessing logic of an application, rule spaces play a crucial role for both the creation of $S \& R$ rules through business operators and the integration of $S \& R$ rules with the event-based infrastructure of an application, in a way that is transparent for end users.

Creating S\&R rules: In the proposed architecture, rule spaces form the basic workspaces for business operators: Each S\&R rule is created within a rule space, from the business-level building blocks of that rule space. With rule spaces grouping eventprocessing logic that makes sense concerning a certain organiza- tional task, business operators are thereby confronted with taskrelevant building blocks only, which facilitates a quick, secure and fail-safe rule creation process. Rule spaces eventually serve as the primary unit for user rights management as they can be assigned to business operators depending on their specific skills and functions within a company.

Executing S\&R rules: Each rule space is assigned to a collection of rule services, which are then said to "host" the given rule space. During run time, all S\&R rules that are created within a rule space are implicitly assigned to all hosting rule services; i.e., whenever a business operator creates a rule, this rule is automatically and transparently applied in appropriate parts of an underlying event-processing map. Accordingly, while the creation of a rule space and the grouping of building blocks is up to rule managers, the assignment of rule spaces to appropriately configured rule services lies in the responsibility of system operators. Both tasks are performed using the Modeling Studio.

In accordance with the overall role of S\&R rules as sketched in Figure 2 - setting up on an event-based abstraction of underlying source systems - a rule space may now be considered as setting up on an event-based abstraction of a certain "aspect" of a business environment. Which parts of a source system actually belong to such aspect is defined by system operators, through the specific event-processing logic that precedes the concerned rule service(s) in the application's event-processing maps. Figure 11 illustrates the described role of rule spaces in UC4 Decision.

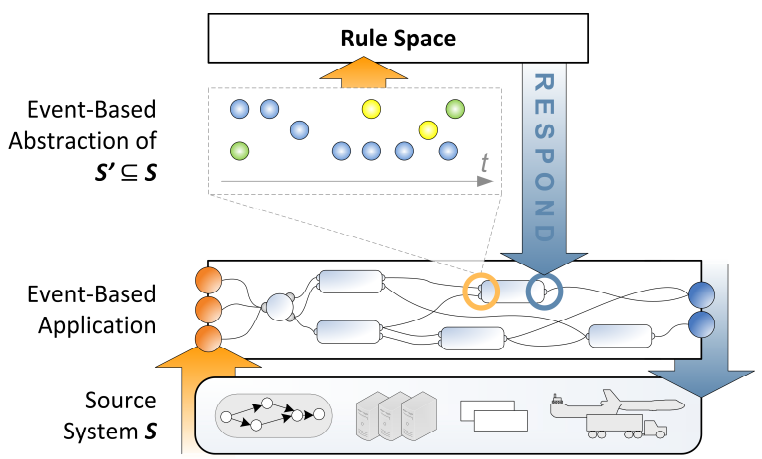

Figure 11. Rule spaces in a Decision application. 


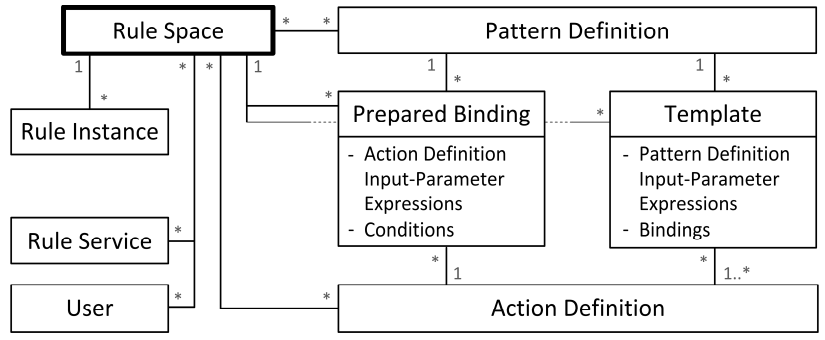

Figure 12. Rule space meta-model.

Figure 12 shows the meta-model for rules spaces. Besides associating pattern and action definitions, rule instances, rule services and authorized user accounts, rule spaces provide additional mechanisms for guiding the work of business operators through collections of templates and prepared bindings:

Templates are structurally equivalent to rule instances, however, may leave open input-parameter expressions for both the pattern and all action definitions. To create a rule from a template, a user simply defines the still missing input-parameter expressions, if any. Rule managers will typically provide templates for rule logic that is required frequently, with equal or similar configuration.

Prepared bindings enable rule managers to prepare associations between pattern and action definitions which are considered as meaningful and/or commonly requested in practical use cases. When creating a rule based on a pattern definition $p$, all prepared bindings for $p$ are suggested to the business operator as being particularly suited in response to the given event situation; whether or not "non-prepared" action definitions are available depends on the specific authorizations of a business operator.

\section{IMPLEMENTATION ARCHITECTURE}

Over the past months, the presented approach to user-oriented rule management has been implemented as an extension to UC4 Decision's existing event processing and rule authorizing facilities. Figure 13 sketches the implementation architecture from a highlevel perspective. On the data layer of the architecture, XMLbased application descriptions define the static structure of the various Decision applications in an installation; among others, such definitions include an application's event-processing map, rule-, pattern- and action definitions, as well as its rule spaces. Each application description eventually refers to an application database, containing all data required during the execution of an application. Apart from active and historic event and correlation data, these include the present set of rule instances.

On the back-end layer, a collection of executor nodes is responsible for actually carrying out the event processing in a distributed execution environment. Infrastructural rules are retrieved from the application description during the deployment of an application. To support hot deployment of business logic, rule instances are retrieved from the application database at regular time intervals. For this purpose, each executor hosts a dedicated rule-instance monitor, which distributes updates to an application's rule services depending on the concerned rule spaces. Note that both infrastructural rules and $\mathrm{S} \& \mathrm{R}$ rules are, essentially, based on decision graphs, which allows using one and the same evaluation mechanism. While the separation between pattern detection and reaction logic in $S \& R$ rules requires some special handling, this has no relevant impact on overall event-processing performance.

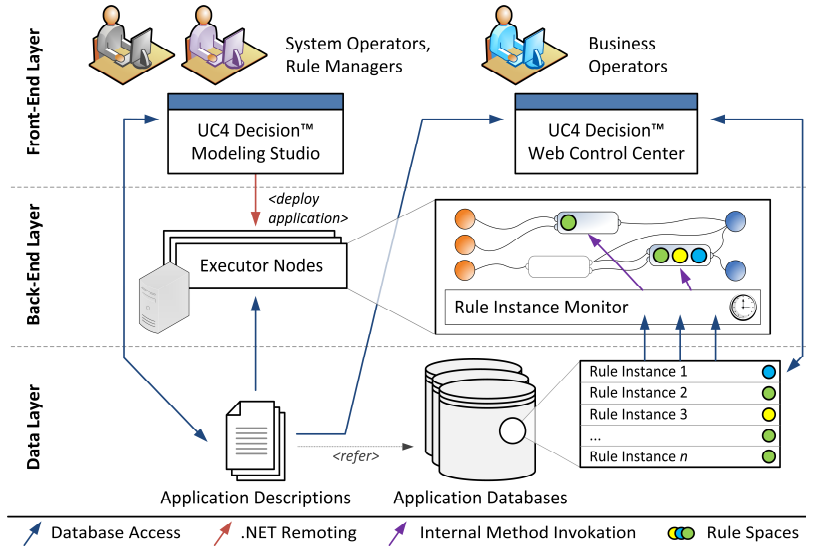

Figure 13. Implementation architecture.

On the front-end layer, tailored interfaces are provided to the different user groups of an application. UC4 Decision Modeling Studio is a .NET-based desktop application that provides power users with sophisticated facilities for creating and modeling Decision applications. Providing editors for rule-, pattern- and action definitions as well as rule spaces, the Modeling Studio is used by system operators as well as rule managers; while former are granted full access to an application, latter are authorized for creating and updating pattern- and action definitions only. Rule instances are eventually administrated via an easy-to-use, wizardbased Web Control Center as shown in Figure 10. While building blocks are retrieved from the application description, the interface does not provide any access to the event-based infrastructure of an application, which allows it to remain simple and focused on high-level decision-making. The Web Control Center has been implemented as an AJAX-based browser application using Google Web Toolkit (GWT).

\section{CONCLUSION}

Event-pattern rules of the form "if pattern, then action(s)" are a key element of Complex Event Processing (CEP) applications and showed to be useful for tasks across different conceptual layers of a systems: On the level of low-level (pre-)processing and integration logic, rules could be used to continuously filter, transform and aggregate events as emerging from the source system. On the level of high-level business logic, rules allow detecting exceptional business situations and triggering counteractions in near real time. We experienced that depending on their particular function in an application, rules are typically associated with different user groups within a company, each having its specific skills, competences and responsibilities. We thus claim that the potential of CEP for building agile business applications can only be unleashed if platforms become manageable and usable within the organizational framework conditions of an enterprise.

In this paper, we presented a novel rule-management framework for CEP applications that caters to the needs of technical-versed power users as well as business users. Technical experts model event-pattern rules in parallel and fully integrated with the others elements of an event-processing application. Business users assemble business logic from prepared, easy-to-use building blocks via a simplified, wizard-based web interface in a workflow that fully abstracts from underlying complexity. 
Since implemented as part of UC4 Decision, the presented framework has successfully been set up at customers from different business domains. Experience from these projects confirmed that a separation into infrastructural and Sense-and-Respond rule management is particularly useful when the high-level business logic of an application shall be administrated by end users with restricted technical skills, and/or changes frequently. Here, significant efficiency enhancements could be achieved for both the customers' IT departments - which may now focus on maintaining the low-level processing logic of an application - and the involved domain experts, which may now administrate their rules by themselves, a straightforward and fail-safe manner.

Experience also showed, however, that in comparison to less flexible solutions that are based on rule definitions only, setting up a full-fledged rule-management system requires considerable additional efforts, e.g., for identifying event-processing logic that qualifies as a "building block", abstracting from such logic through input and output parameter, and defining and configuring rule spaces. Such efforts may not be justified in smaller installations or if all users have sufficient skills anyway. It is essential to note, though, that the proposed framework provides full support for both kinds of architectures; if a separate S\&R layer is not rewarding, the entire functionality can just as well be implemented as part of the event-based infrastructure.

Resulting in applications that are inherently generic and adaptable to the current processing needs of an enterprise through $S \& R$ rules, existing projects eventually showed that our framework supports the definition of "standardized" CEP solutions, which can be offered to multiple customers with similar basic processing requirements and business environments. Customers can then benefit from the typical advantages of commercial off-the-shelf (COTS) software, including reduced costs, rapid deployment, well-tested and documented functionality, standardized updates, etc. Following from a large project from the workload automation domain, many of the created pattern and action definitions evolved into a so-called solution template, which by itself could be delivered to several other customers with minimum customerspecific adaptations, if any.

\section{REFERENCES}

[1] Abadi, D. J., Carney, D., Cetintemel, U., Cherniack, M., Convey, C., Lee, S., Stonebraker, M., Tatbul, N., and Zdonik, S. 2003. Aurora: A new model and architecture for data stream management. VLDB Journal, 12(2): 120-139.

[2] Adi, A., Botzer, D., Nechushtai, G., and Sharon, G. 2006. Complex Event Processing for financial services. In Proceedings of the IEEE Services Computing Workshops (SCW'06). IEEE Computer Society, Washington, DC.

[3] Adi, A. and Etzion, O. 2004. AMIT - The situation manager. VLDB Journal, 13(2): 177-203.

[4] Ammon, R. v., Silberbauer, C., and Wolff, C. 2007. Domain specific reference models for event patterns. In VIP Symposia on Internet related research with elements of $M+I+T++$.

[5] Chandy, K. M. and Schulte W. R. 2010. Event Processing: Designing IT Systems for Agile Companies. McGraw-Hill, New York, NY.

[6] Chakravarthy, S. and Jiang, Q. C. 2009. Stream Data Processing. Springer, Berlin.
[7] Demers, A., Gehrke, J., Panda, B., Riedewald, M., Sharma, V., and White, W. 2007. Cayuga: A general purpose event monitoring system. In Proceedings of the International Conference on Innovative Data Systems Research, 412-422.

[8] Eckert, M. and Bry, F. 2010. Rule-based composite event queries: the language $\mathrm{XChange}^{\mathrm{EQ}}$ and its semantics. Knowledge and Information Systems, Springer, London.

[9] Esper, http://esper.sourceforge.net

[10] Etzion, O. and Niblett, P. 2010. Event Processing in Action. Manning Publications, Stamfort, CT.

[11] Gualtieri, M. and Rymer, J.R. 2009. The Forrester Wave ${ }^{\mathrm{TM}}$ : Complex Event Processing (CEP) Platforms, Q3 2009. Forrester Research, Cambridge, MA.

[12] Hinze, A., Sachs, K., and Buchmann, A. 2009. Event-based applications and enabling technologies. In Proceedings of the Third ACM International Conference on Distributed EventBased Systems (DEBS'09). ACM, New York, NY.

[13] Kellner, I. and Fiege, L. 2009. Viewpoints in complex event processing: industrial experience report. In Proceedings of the Third ACM International Conference on Distributed Event-Based Systems (DEBS'09). ACM, New York, NY.

[14] Luckham, D. 2002. The Power of Events. Addison-Wesley, Boston, MA.

[15] Luckham, D. 2006. What's the Difference Between ESP and CEP? http://complexevents.com/?p=103

[16] Paschke, A. and Vincent, P. 2009. A reference architecture for Event Processing. In Proceedings of the Third ACM International Conference on Distributed Event-Based Systems (DEBS'09). ACM, New York, NY.

[17] Paschke, A., Kozlenkov, A., and Boley, H. 2007. A homogenous reaction rules language for Complex Event Processing. In Proceedings of the $2^{\text {nd }}$ International Workshop on Event Drive Architecture and Event Processing Systems (EDA-PS'07)

[18] Rozsnyai, S., Obweger, H., and Schiefer, J. 2008. Event access expressions: A business user language for analyzing event streams. In Proceedings of the $25^{\text {th }}$ International Conference on Advanced Information Networking and Applications (AINA'11). To be published.

[19] Schiefer, J., Obweger, H., and Suntinger, M. 2009. Correlating Business Events for Event-Triggered Rules. In RuleML'09, 67-81. Springer, Berlin.

[20] Schiefer, J., Rozsnyai, S., Rauscher, C., and Saurer, G. 2007. Event-driven rules for sensing and responding to business situations. In Proceedings of the International Conference on Distributed Event-Based System (DEBS'07), 198-205.

[21] Seiriö, M. and Berndtsson, M. 2005. Design and Implementation of an ECA Rule Markup Language. In RuleML'05, 98-112. Springer, Berlin.

[22] Sen, S. and Stojanovic, N. 2010. GRUVe: A methodology for Complex Event Processing life cycle management. In International Conference on Advanced Information Systems Engineering (CAiSE'10). Springer, Berlin.

[23] Turchin, Y., Gal, A., and Wasserkrug, S. 2009. Tuning complex event processing rules using the predictioncorrection paradigm. In Proceedings of the Third ACM International Conference on Distributed Event-Based Systems (DEBS'09). ACM, New York, NY. 